\title{
On the Theory of General Advection
}

\author{
by \\ T. Yamaoka \\ Meteorological Research Institute
}

(Reccived July 20, 1951)

\begin{abstract}
Reflecting on the twenty years' history of the theory of general advection it is pointed out that the subject must be dealt with as a well-defined dynamical problem, in order that that a definite unobjectionable theory might be developed. In Section 2, after making clear the process of advection and defining accurately the quantities concerned, the fundamental formulas of advection are derived, which are more comprehensive than those ever published and the meanings of several previous theories are explained. Section 3 deals with the contribution of thermal. effect to the advection and Section 4 the effect of the discontinuous surface. In Section 5 new forms of advection formulas are presented, which are useful for practical purposes.
\end{abstract}

\section{Introduction}

As is well known, when Rossby's formula of general advection is applied to the observed pressure changes of the atmosphere, the advection function often reaches in a preiod of a day or two a value much larger than $10 \mathrm{~mm} \mathrm{Hg}$ even in the stratosphere [1]. It is doubtful whether we can consider so intense an advection as really taking place above the tropopause. Ertel derived another formula [2], but this was not correct and its inconsistency was later pointed out by him [3]. Although Rossby's formula is exact, it is based on the restricting assumptions that the process is non-divergent and adiabatic (or polytropic). Further since his method of deduction was founded on the result of his theory of special advection [4], it was not straight for ward. Ertel and Kuo [5] used this method for deriving their respective formulas of advection. Kuo's formula

$$
\delta z=-\frac{1}{\gamma} \int_{0}^{z} \frac{\delta \pi}{p} d z-\frac{1}{g} \int_{0}^{z} \frac{1}{\rho} \frac{d \delta \pi}{d z} d z
$$

where $\delta z$ represents the vertical displacement of the layer due to advection, does not ogree with the facts. For insance if we assume the air as adiabatically incompressible $(\gamma \rightarrow \infty)$, the vertical displacement $\delta z$ must evidently vanish for any horizonsal advection, while according to the above equation $\delta z$ may have a value different from zero owing to the second integral, when a horizontal advection of the air with different density takes place in any stratum. 
Philipps [6] and Ertel [3] obtained the formal expressions of the advection including hori-ontal divergence, Dufour treated the problem in a general case [7]. His method of deduction does not follow Rossby's line and corresponds better to the dynamical nature of the advection problem.*

The problem of advection must be dealt with as a well-defined dynamical one, and a definite unobjectionable theory is to be developed. In the past, however, the progress has not been steady since Rossby's pioneer works. The reason thereof seems to be that the concepts concerning the advection process have not been clearly defined, the methods of analysis used hitherto were too special and these circumstances gave rise to some confussions in this problem.

In view of the above situation it is attempted in Section 2 of the present paper to make clear the process of advection, to give precise definitions to the variations of the related meteorological elements and then to derive the fundamental formulas of advection which are more comprehensive than those of the previous theories. By means of these equations it is possible to explain the meanings of the formulas of Rossby, Ertel, Philipps and Dufour. In Section 3 the fundamental formulas are applied to the advection subject to non-polytropic heating or cooling such as takes place in the lowest atmosphere. The older theories, being confined to the case of adiabatic or polytropic changes, were not applicable to these cases. In Section 4 we consider the influence of discontinuous surfaces upon the effect of advection. In Section 5 a new form of advection formula is presented, which is suitable for the upper air analysis and also by means of which the picture of advection is. easily grasped from the data of upper air soundings.

\section{Derivation of the fundamental formulas}

Any advection in atmosphere will be represented as follows. In Fig. 1 the state of an air column $\mathrm{A}$ of unit cross section prior to an advection is denoted by $\mathrm{A}$. Let the state of the air column B, which has replaced $A$ by the advection, be denoted by B 3. Before the advection $B$ is generally neither arranged in a vertical column nor hasa uniform cross section, as is shown by B1. Consequently the condition of statical equilibrium need not be satisfied in the state B 1. Now let us assume that the air $B$ is made to pass imaginarily through an arbitrarily se-

* The accroisement $\delta_{e} p$ de la pression hydrostatique

$$
\hat{\delta}_{e} p=\int_{z}^{\infty} y \frac{\delta_{e} d m}{d s}==\int_{z}^{\infty} g \rho\left(\zeta_{e}+\sigma_{c}+\frac{\delta_{e} \rho}{\rho}\right) d z
$$

as defined by Dufour is not the pressure increment. The correct form of the latter must be

$$
\delta_{e} p^{\prime}=\int_{z}^{\infty} g \frac{\delta_{e} d m}{d s+\delta_{e} d s}=\int_{z}^{\infty} g \rho\left(\zeta_{e}+\frac{\delta_{e} o}{\rho}\right) d z
$$

$\delta_{e} p$ coincides with $\delta_{e} p^{\prime}$ only when $\sigma_{e}=\frac{\delta_{e} d s}{d s}=0$.

Practically among the special cases which Dulour dealt with as applications of his gencral formulas, there is none in which $\sigma_{e}$ does not vanish or plays a rôle significant by itself. When any case would be considered in which $\sigma_{e}$ is effective, $\delta_{e} p$ must be correctly treated in conformity with its definition. 
lected state B2 on the way from B1 to B 3 . As the state B 2 generally is not a state actually passed by $\mathrm{B}$, it may be necessary to apply some imaginary forces to $\mathrm{B}$. We suppose, these imaginary forces are applied by inserting imaginary screens between each thin layer of B to compensate the differences of air pressures between the upper layer and the lower of each screen and by using imaginary vertical walls to keep the initial cross sections of the layers. After bringing $\mathrm{B}$ from $\mathrm{B} 1$ to $\mathrm{B} 2$ we renove these additional forces. The vertical displacements and the horirontal divergences (or convergences) will ensue, so that the final air pressure of each laver will be balanced with the final weight of the upper air column per unit cross section. Thus B will reach the state B3. From the nature of our problem it is clear that we do
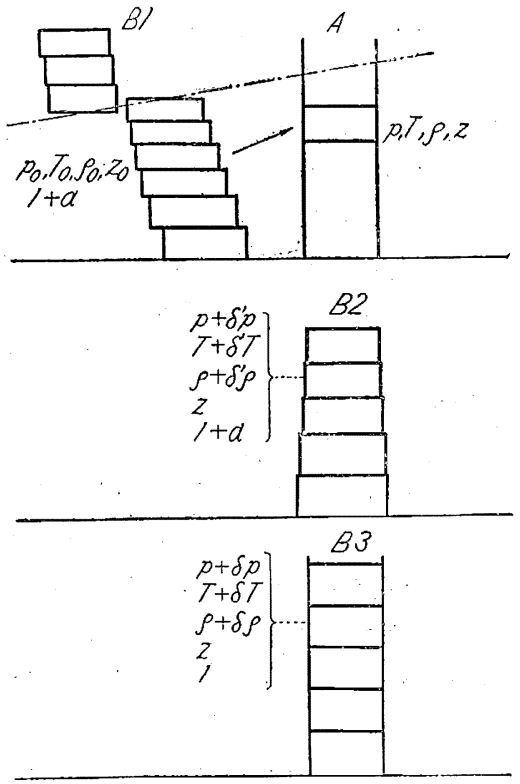

Fig. 1 Scheme of advection not lose any generality by considering as above:

Let the atmospheric pressures, temperatures, densities, cross sections and altitudes of the corresponding layers of the air columns A and B be designated as shown in the following table and at the same time the seven variations $\delta^{\prime} p, \delta^{\prime} T$, $\cdots . ., \delta z$ and the horizontal divergence $-\alpha$ be defined thereby. Here the "corresponding layers of $\mathrm{A}$ and $\mathrm{B}$ " indicate the pair of the layers of $\mathrm{A}$ and $\mathrm{B}$, the latter of which is brought to the position of the former in the state B2.

\begin{tabular}{cccc} 
A & B1 & B2 & B3 \\
\hline$p$ & $p_{0}$ & $p+\delta^{\prime} p$ & $p+\delta p$ \\
$T$ & $T_{0}^{\prime}$ & $T+\delta^{\prime} T$ & $T+\delta T^{\prime}$ \\
$\rho$ & $\rho_{0}$ & $\rho+\delta^{\prime} \rho$ & $\rho+\delta \rho$ \\
1 & $1+\alpha$ & $1+\alpha$ & 1 \\
$z$ & $z_{0}$ & $z$ & $z+\delta z$
\end{tabular}

Note: Notations are used regardless of those used in Section 1.
When we aim at the individual change of each layer of $B$, the variations during the process $B 1 \rightarrow B 3$,

$$
p+\delta p-p_{0} \quad \text { and } z+\delta z-z_{0}
$$

may be called the advection function and the vertical displacement respectively. Or alternatively when we take up the difference between the final state of each layer of $\mathrm{B}$ and the state of the corresponding layer of $A$, the variations

$$
p+\delta p-\delta p=\delta p \quad \text { and } z+\delta z-\delta z=\delta z
$$

may be called the advection function and the vertical displacement respectively.

In the present paper no restriction is assumed about the physical. change to which the air of $\mathrm{B}$ is subject during the advection. But it will be convenient to deal with the general case of non-polytropic thermal action separately in Section 3.

First we consider the change of a layer of B from B 2 to B 3. We have

$$
\frac{1}{\gamma} \frac{\delta p-\delta^{\prime} p}{p}=\frac{\delta \rho-\delta^{\prime} \rho}{\rho}, \quad \text { Condition of physical change }
$$

$$
\frac{\delta \rho-\delta^{\prime} \rho}{\rho}=\alpha-\frac{d \delta z}{d z}, \quad \text { Equation of continuity }
$$


where

$$
-\alpha=\frac{\partial \delta x}{\partial x}+\frac{\partial \delta y}{\partial y}
$$

For dry adiabatic change $\gamma=c_{p} / c_{v}$ and for saturation adiabatic change

$$
\gamma=\frac{\Gamma d}{\Gamma d-\kappa \Gamma v m} \fallingdotseq \frac{\Gamma d}{\Gamma d-\Gamma \kappa m},
$$

where $\Gamma d$ and $\Gamma m$ are the dry and the saturated adiabatic lapse rate respectively, $\Gamma v m$ the saturated adiabatic lapse rate of the virtual temperature and $\kappa=\left(c_{p}-c_{v}\right) / c_{p}{ }^{*}$

By the condition (1) the equation of continuity becomes

$$
\frac{d \delta z}{d z}=-\frac{\delta p-\delta^{\prime} p}{\gamma p}+\alpha .
$$

Summing up the increments of the thickness $d(\delta z)$ for all the layers below the layer in consideration we obtain the vertical displacement

$$
\delta z=-\int_{0}^{z}\left(\frac{\delta p-\delta^{\prime} p}{\gamma p}-\alpha\right) d z \text {. }
$$

This formula holds good even if $\gamma$ may vary with the height as in the case of saturated adiabatic change.

Now let $\partial p$. designate the local variation of $p$ at a fixed level $z$. As $\partial p$ is the difference of the pressure of B 3 and that of A both at the level $\approx$, we have obviously

$$
\delta p=\partial p+\frac{\partial p}{\partial z} \delta z
$$

Hence

$$
\partial p=\delta p-\frac{\partial p}{\partial z} \int_{0}^{z}\left(\frac{\delta p-\delta^{\prime} p}{\gamma p}-\alpha\right) d \varepsilon
$$

The equations (5) and (7) give $\delta z$ and $\partial p$ respectively expressed by the advection function $\delta p$.

To express $\delta z$ and $\delta p$ by the local pressure change we eliminate $\delta p$ from (4) and (6) and obtain

$$
\frac{d \delta z}{d z}+\frac{1}{r p} \frac{\partial p}{\partial z} \delta z-\alpha+\frac{\partial p-\delta^{\prime} p}{p}=0
$$

which is integrable in simple form, when $r$ is independent of $z$ as in the case of dry adiabatic change. Thus

$$
\delta z=-\frac{1}{p^{\frac{1}{r}}} \int_{0}^{z} p^{-\frac{1}{r}}\left(\frac{\partial p-\delta^{\prime} p}{r p}-\alpha\right) d z .
$$

* To derive the equation (3) we differentiate the equation of gas $p=R \rho T_{v}$, where $T_{v}$ is the virtual temperature, along the saturation adiabatic:

On the other hand

$$
\frac{d \rho}{\rho}-\frac{d p}{p}=-\frac{d T_{v}}{T_{v}^{\prime}}=\frac{\Gamma^{v} v m}{T_{v}^{\prime}} d z
$$

$$
\frac{d p}{p}=-\frac{g \rho}{p} d z=-\frac{g}{R T_{v}} d z=-\frac{A g}{c_{p}-c_{v}} \frac{d z}{T_{v}}=-\frac{\Gamma d}{\kappa} \frac{d z}{T_{v}} .
$$

Taking the ratios of both members of these equations and defining $\gamma$ by the equation $\frac{1}{\gamma}=\frac{p}{\rho} \frac{d \rho}{d p}$, which is equivalent to (1), we have

$$
\frac{1}{\gamma}-1=-\kappa \frac{\Gamma v m}{\Gamma^{\prime} d}
$$


The advection function $\delta p$ is obtained by substituting for $\delta z$ of (6) from (9),

The above results give the formulas of advection more comprehensive than those ever published. The equations (5), (7) and (9) include the formulas of Rossby, Ertel, Philipps and Dufour as special cases. Before proceeding further, let us consider what the above equations mean. All these equations concern only the reations among the variations which take place during the imaginary process $\mathrm{B} 2$ $\rightarrow$ B 3. In fact the equation (5) or (4) is the relation among the three variations $\delta p \rightarrow \delta^{\prime} p, \alpha$ and $\delta z$ during the process B $2 \rightarrow$ B 3 . The equation (9) or (8) is the relation among $\partial p-\delta^{\prime} p, \alpha$ and $\delta z$, and $\partial p-\delta^{\prime} p$ is the the local pressure change between $B 2$ an $B 3$ as will be easily seen. The equation (7) or rather (6) is equiva ent to

$$
\delta p-\delta^{\prime} p=\partial p-\delta^{\prime} p+\frac{\partial p}{\partial z} \delta 2 .
$$

and the three variations $\delta p-\delta^{\prime} p, \partial p-\delta^{\prime} p$ and $\delta z$ are all quantities related to the process $\mathrm{B} 2 \rightarrow \mathrm{B} 3$. Thus these equations concern neither the actual change state $\mathrm{B} 1$ $\rightarrow \mathrm{B} 3$ or $\mathrm{A} \rightarrow \mathrm{B} 3$. As, whatever intermediate state we may assume, the final state B 3 is determinate depending only on the initial state B1, $p+\delta p, s+\delta z, \alpha$ etc. are independent of the state B 2 . Therefore if we assume another intermediate state, that is, take other values for $p$, z etc., the variations $\delta p$, $\delta z$ etc. will necessarily change. (At the same time the variations $\delta^{\prime} p, \cdots \cdots$ belonging to the state B 2 will have different values) Thus the variations $\delta p$ and $\delta z$ given by (5), (7) and (9) have no actual meaning unless some suitable conditions are imposed for the state B 2 .

One of the simplest and natural assumptions for selecting the state B 2 is the following. In order that the variation $\delta$ a given by (5) or (9) may represent the individual vertical displacement $z+\delta z-z_{0}$, we must put $z=z_{0}$, in other words, B2 must be such as is obtained by moving each layer of B 1 horizontally. Then the volume of each layer does not vary, because both the thickness and the cross section of each layer are kept constant during the change $\mathrm{B} 1 \rightarrow \mathrm{B} 2$. Consequently, in so far as the adiabatic or po'ytropic change is assumed as is stated by (1), the density, the pressure and the temperature do not vary. Thus $\rho+\delta^{\prime} \rho=\rho_{0}, p+\delta^{\prime} p=p_{0}$ $T+\delta^{\prime} T^{\prime}=T_{0}$. Hence, denoting the horis ontal displacement by $v=(\delta x, \delta y)$, we obtain

$$
\delta^{\prime} p=p_{0}-p=-\frac{\partial p}{\partial 2} \delta x-\frac{\partial p}{\partial y} \delta y=-v \operatorname{grad}_{2} p(=\Delta p),
$$

which is the deviation of the gradient wind and will be designated by $\Delta p$. It is easily seen that, substituting for $\delta^{\prime} p$ in (9) from (10), the equation (9) becomes Ertel's formula

$$
\delta p=\partial p+\frac{y}{R \theta} \int_{0}^{z} \frac{\theta}{\gamma} T^{\prime} \partial p d z+\frac{g}{\theta} \int_{0}^{z}\left\{\frac{\partial(p \delta x \theta)}{\partial x}+\frac{\partial(\rho \delta y \theta)}{\partial y}\right\} d z,
$$

where $\theta$ is the potential temperature, as well as Philipps' formula

Advection function for unit time $=\frac{\partial p}{\partial t}+\frac{g p^{\kappa}}{R \bar{T}} \int_{0}^{z} p^{\frac{1}{r}}\left\{\operatorname{div}: \boldsymbol{v}+\frac{1}{r p}\left[\frac{\partial p}{\partial t}+\left(\operatorname{vgrad}_{2} p\right)\right]\right\} d \boldsymbol{x}$. 
If we neglect both the horizontal divergence $-\alpha$ and the deviation of gradient wind $\Delta p$ (generally $\delta^{\prime} p$ ), (7) and (9) are reduced to Rossby's formulas. But these terms are not necessarily negligible as will be shown in Section. 5. *

Now the above equations do not constitute by themselves a complete set of equations for determining all the variations mentioned above. We have defined B 3 as the state in which all the screens between the layers have been removed and the pressure of each layer is in equilibrium with the weight of the upper air column per unit cross section. However, in the analysis described above we have not yet taken into account this condition of statical equilibrium. Hence in the equations obtained above we could even consider the screens as not yet removed and assume some imaginary forces as still acting. In such a case the equation of statical equilibrium would be modified as

$$
d p=-g \rho d z+F d z,
$$

where $F$ denotes the residual force per unit thickness of the column. Now in order to express that the statical equilibrium is realized in the state $B 3$ as well as in $A$, we must put

$$
\begin{aligned}
\text { (a) } & \mathrm{A}: & p=g \int_{z}^{\infty} \rho d z, \\
\text { (b) } & \mathrm{B}: & p+\delta p=g \int_{z}^{\infty}(\rho+\delta \rho) d(z+\delta z) .
\end{aligned}
$$

Subtracting the former equation from the latter, we get, with consideration to (2),

$$
\begin{gathered}
\delta p=g \int_{z}^{\infty}\left(\delta \rho+\rho \frac{d \delta z}{d z}\right) d z=g \int_{z}^{\infty}\left(\delta^{\prime} \rho+\rho \alpha\right) d z, \\
\frac{d \delta p}{d z}=-g\left(\delta \rho+\rho \frac{d \delta z}{d z}\right)=-g\left(\delta^{\prime} \rho+\rho \alpha\right) .
\end{gathered}
$$

In summary, if the initial conditions of an advection are known, the values of $\delta^{\prime} p, \delta^{\prime} \gamma, \delta^{\prime} \rho, \alpha$ and $z_{0}$ will be determined as functions of $z$ by assuming a suitable intermediate state B 2. The advection function $\delta p$ is given by (11) and then $\delta z$ and $\partial p$ are obtained by (5) and (6) or (7) respectively. $\delta p$ and $\delta I '$ will be determined by (1) and the variation of the equation of gas

* For explaining Dufour's treatinent we return to the beginning of this section. The cross. section of the state $B 3$ can be, ceteris paribus, taken to be $1+\beta$ instead of unity. Then the equation of continuity (2) becomes

$$
\frac{d \delta z}{d z}=-\frac{\partial \rho-\delta^{\prime} \rho}{\rho}-(\alpha-\beta) .
$$

If we write $z_{0}=z+\delta^{\circ} z, \delta z-\delta^{\circ} z$ is the vertical displacement of the air of $\mathrm{B}$ due to the process: $\mathrm{I} 1 \rightarrow \mathrm{B} 2$, and the above equation becomes

$$
\frac{d\left(\delta z-\delta^{\circ} z\right)}{d z}=-\frac{\delta \rho-\delta^{\prime} \rho}{\rho}-\frac{d \delta^{\circ} z}{d z}-\alpha+\beta
$$

If we write $\delta z-\delta^{\circ} z \rightarrow \delta z,-\frac{d \delta^{\circ} z}{d z} \rightarrow \zeta_{e}, \delta^{\prime} \rho \rightarrow \delta_{e} \rho, \rightarrow \alpha \rightarrow \sigma_{e}$ and $-\beta \rightarrow \sigma$, we get

$$
\frac{d \delta z}{d z}=-\frac{\delta \rho-\delta_{\epsilon}{ }^{0}}{\rho}+\zeta_{\epsilon}+\sigma_{e}-\sigma,
$$

which is the equation. (7) in Dufour's paper serving as the basis of his theory. 


$$
\frac{\delta p}{p}=\frac{\delta \rho}{\rho}+\frac{\delta T}{T}
$$

When as state $B 2$ we choose one which is obtained by translating B 1 adiabatically and horizontally. $\delta z$ means the individual vertical displacement.

\section{Advection with non-polytropic heating or cooling}

We will consider the advection subject to general thermal action. Denoting by $q$ the heat quantity given to a unit mass of the air, we have by the first law of thermodynamics

$$
\frac{q}{c_{p} T}=\frac{1}{\gamma} \frac{d p}{p}-\frac{d \rho}{\rho}
$$

Putting $q=c_{p} \Delta T$ and applying the avove equation to the process B $2 \rightarrow \mathrm{B} 3$; we wet

$$
\frac{1}{\gamma} \frac{\delta p-\delta^{\prime} p}{p}=\frac{\delta \rho-\delta^{\prime} \rho}{\rho}+\frac{\Delta T}{T^{\prime}}
$$

$\Delta T^{\prime}$ is the increase of temperature which would take place if $q$ were given to the air under the constant atmospheric pressure. The equation (13) is a generalization of (1).

Therefore, as will be seen by comparing this equation with (1), the formulas of the advection subject to the general thermal action will be obtained from the formulas discussed in Section 2 by substituting

$$
\frac{\delta^{\prime} p}{r p}+\frac{\partial^{\prime} T}{T} \quad \text { for } \quad \frac{\delta^{\prime} p}{r p} \text {. }
$$

When the heat quantity $q$ is given to the air in the process $\mathrm{B} 1 \rightarrow \mathrm{B} 2$, the change of the air pressure $p+\delta^{\prime} p-p_{0}$ will be greater by $r p \cdot \frac{q}{c_{p}} T^{\text {, than }}$ that of the adiabatic or polytropic process, according to the equation similar to (13). Hence $\delta^{\prime} p$ will be greater by $r p \cdot \frac{\Delta T}{T}$, in other words $\frac{\delta^{\prime} p}{\gamma p}$ will be greater by $\frac{\Delta T}{T}$.

Hence, independent of whether the thermal action has taken place in $B 1 \rightarrow B 2$ or in $\mathrm{B} 2 \rightarrow \mathrm{B} 3$, the formulas of advection accompanied by the general thermal action can be obtained by making the substitution (14) in the equations (4); (5), (7), (8) and (9). For example generalizations of (5) and (9) are respectively

$$
\begin{aligned}
& \delta_{z}=-\int_{0}^{z}\left(\frac{\delta p-\Delta p}{\gamma p}-\alpha-\frac{\Delta T}{T}\right) d z, \\
& \delta_{z}=-\frac{1}{p^{\prime}} \int_{0}^{z} p^{\frac{1}{r}}\left(\frac{\partial p-\Delta p}{\gamma p}-\alpha-\frac{\Delta T}{T}\right) d z .
\end{aligned}
$$

We can express the heat quantity $q$ or $\Delta T$ by the observed meteorological elements $T^{\prime}$ and $p$ as follows

$$
\frac{\Delta T}{T}=\frac{q}{c_{p} T}=\frac{\delta^{\prime} T-\delta^{\prime} T^{\prime}}{T}-\left(1-\frac{1}{\gamma}\right) \frac{\delta p-\Delta p}{p} .
$$

The relation is obtained by considering the heat quantity $q$ as given during the process B $2 \rightarrow$ B 3 and applying the first law of thermodynamics to the process. 
In summary the order of determining the variations is formally the following: When the heat quantity $q$ (or $\Delta T$ ) is unknown, another datum is necessary. If for instance the local pressure change $\partial p$ is known, $\delta p$ is determined by (11) as before, $\delta z$ is given by (6) and $\Delta T$ by the differentiation of (15) or (16) by $z . \quad \delta T$ and $\delta \rho$ are determined by the equation (13) and the variation of the equation of gas.

\section{The influence of the discontinuous surface}

When there is a discontinuous surface as shown in Fig. 1, we separate the air column into the upper part and the lower of the discontinuous surface and denote

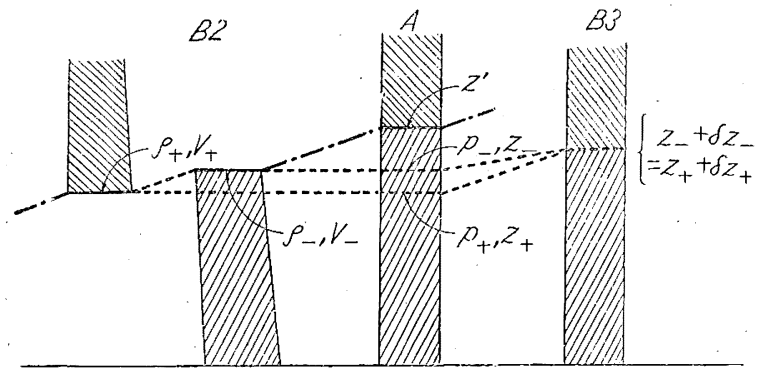

Fig. 2 Analysis of discontinuous surface

$$
\delta z=-\int_{z_{+}}^{z^{i}}(\cdots \cdots) d z+\delta z_{+}
$$

by $\delta z_{\text {_ }}$ the vertical displacement of the top of the lower column. From (15) we have, referring to Fig. 2,

$$
\delta z_{-}=-\int_{0}^{z-}(\cdots \cdots) d z .
$$

As the bottom of the upper column is not fixed but replaced by, say, $\delta z_{+}$, the vertical displacement in the upper column is

Denoting the difference of the vertical displacements at the discontinuous surface by $\delta Z=\delta z_{+}-\delta z_{-}$, we have from the above equations

$$
\left.\delta z=\delta Z-\left\{\int_{0}^{z}(\cdots \cdots) d z+\int_{z_{+}}^{z}-\cdots \cdots\right) d z\right\} .
$$

As the second integral is negligible against the first, we obtain for the vertical displacement in the upper column

$$
\delta z=\delta Z-\int_{0}^{z}\left\{\frac{\delta p-\Delta p}{\gamma p}-\alpha-\frac{\Delta T}{T}\right\} d z .
$$

Considering $z_{-}+\delta z_{-}=z_{+}+\delta z_{+}$and designating the discontinuous surface by $z=H(x, y)$ we have

$$
\delta Z=\delta z_{+}-\delta z_{-}=z_{-}-z_{+}=\Delta v \operatorname{grad}_{2} H,
$$

where $\Delta v$ is the difference of the horizontal disp'acements above and below the discontinuous surface. For the lower column the equation (15) holds instead of (18). $\Delta p$ is given by (10).

Next the equations of statical equilibrium (11) and (12) must be extended. Above the discontinuous surface the relation (11) holds. It will be shown that below the discntiruous surface the relation (22) below holds. The advection function at the base of the upper, column is by (11).

$$
\delta p_{+}=g \int_{z_{+}}^{\infty}\left(\delta^{\prime} \rho+\rho \alpha\right) d z .
$$

Denoting the advection function at the top of the lower column by $\delta p_{-}$and considering $p_{+}+\delta p_{+}=p_{-}+\delta p_{-}$, we have 


$$
\begin{aligned}
& p_{+}-p_{-}=\delta p_{-}-\delta p_{+}=g \rho \delta Z=g \rho \Delta v \operatorname{grad}_{2} H\left(=\delta I^{\prime}\right), \\
& \delta p_{-}=\delta P+\delta p_{+} \text {, }
\end{aligned}
$$

where $\delta P$ is defined by (20). By (21) we can easily obtain

$$
\delta p=\delta P+g \int_{z}^{\infty}\left(\delta^{\prime} \rho+\rho \alpha\right) d z
$$

as the generalization of (11) which holds for the lower column. The relation (12) is applicable both above and below the discontinuous surface.

For reference let us consider the meaning of the singuläre Advektion dicsussed by Ertel [8] using our notations. The equations of statical equilibrium for $A$ and B1 are respectively

$$
p=g \int_{z}^{\infty} \rho d z \quad \text { and } \quad p_{0}=g \int_{z}^{\infty} \rho_{0} d z \text {. }
$$

When the layer $z$ is below the discontinuous surface, the integrand $\rho_{0}-\rho$ of the pressure difference $p_{0}-p=g \int_{z}^{\infty}\left(\rho_{0}-\rho\right) d z$ has a finite value between $z_{+}$and $z^{\prime}$ at the discontinuous surface and a specical attention is required for the evaluation of the integral. Denoting the singuläre Advektion by $\delta P$ s we have, as is easily seen in Fig. 2,

Writing

$$
\delta P_{s}=g \int_{z_{+}}^{z^{\prime}}\left(\rho_{0}-\rho\right) d z=g\left\{\rho_{+}\left(z^{\prime}-z_{+}\right)-\rho_{-}\left(z^{\prime}-z_{-}\right)\right\} .
$$

$$
\rho_{+} \boldsymbol{v}_{+}-\rho . \boldsymbol{v}_{-}=\Delta(\rho \boldsymbol{v})
$$

the above equation becomes

$$
\delta P_{s}=g \Delta(\mid \boldsymbol{v}) \operatorname{grad}_{2} H,
$$

which is the relation given by Ertel.

\section{The advection formulas with the atmospheric pressure as the parameter}

Let $\partial T^{\prime}$ indicate the change of temperature at a costant pressure due to an advection. In other words $\partial T^{\prime}$ is the difference of the temperatures along an isobar on the adiabatic diagram before and after the advection. Therefore $\partial T^{\prime}$ can be directly read on the diagram.

From the equation (12) and the equation of statical eqilibrium, which are applicable both below and above the discontinuous surface, we have

$$
\frac{d \delta p}{d p}=\frac{\delta^{\prime} \rho}{\rho}+\alpha
$$

which becomes, by using the variation of the equation of gas,

$$
\frac{d \delta p}{d p}=-\frac{\delta^{\prime} T}{T^{\prime}}+\alpha-\frac{\delta^{\prime} p}{p} \text {. }
$$

On the other hand the equation of physical change is

$$
\frac{\gamma-1}{\gamma} \frac{\delta p-\delta^{\prime} p}{p}=\frac{\delta^{\prime} T^{\prime}-\delta^{\prime} T}{T}
$$

and by the comparison of $\mathrm{A}$ and $\mathrm{B} 3$ we have

$$
\partial T=\delta T-\frac{\partial T}{\partial T} \delta p .
$$


Eliminating $\delta T$ and $\delta^{\prime} T$ from the three equations we obtain

$$
\frac{d \delta p}{d p}+\left(\frac{1}{T} \frac{\partial T^{r}}{\partial p}-\frac{\kappa}{p}\right) \delta p+\frac{\partial T^{\prime}}{T}-\alpha-\frac{\delta^{\prime} p}{\gamma p}=0
$$

Integrating (25) by $p$ on the assumption of dry adiabatic change i.e. $(\gamma-1) / r=\kappa$ and substituting

$$
\frac{\Delta p}{\gamma p}+\frac{\Delta T}{T} \quad \text { for } \quad \frac{\delta^{\prime} p}{\gamma p}
$$

according to (10) and (14), we finally abtain

$$
\delta p=\frac{1}{\theta}\left\{\theta_{s} \delta p_{s}+\int_{p}^{p_{s}} \theta\left(\frac{\partial T-\Delta T}{T}-\alpha-\frac{\Delta p}{\gamma p}\right) d p\right\},
$$

where the suffix $s$ indicates the values at the surface. For a level above the discontinuous surface, integrating (24) from there to the level in consideration $(p)$ and taking into account the boundary condition (21), we obtain

$$
\theta \delta p=\theta_{s} \delta p_{s}-\theta_{+} \delta P+\int_{p}^{p_{s}} \theta\left(\frac{\partial T-\Delta T}{T}-\alpha-\frac{\Delta p}{\gamma p}\right) d p .
$$

Here $\theta_{+}$. is the value of the potential temperature at the upper boundary of the discontinuous surface. Alternatively the value at the lower boundary $\theta_{-}$may be used.

In the formula (25) the four factors which contribute to the formation of advection function in a direct or a indirect way appear all in simpie and similar forms and are connected to one another in the form of summation, so that one can easily compare the relative importance of these factors at actual advection analysis. Although the term. $\Delta T / T$ and possibly $\Delta p / \gamma p$ will rapidly decrease with the height, it should be observed that the contribution of $\theta d p$ to the integral is larger in the lower atmosphere.

By means of the formulas (25) or (26) the advection function $\delta p$ can be easily accurately calculated or estimated without conducting the height computation, while it is indispensable when we use the formulas discussed in the previous sections. It will be useful to mention a formula which enables us to find graphically the advection-free regions on the thermodynamic diagram. For the sake of simplicity let us neglect the terms of horizontal divergence and thermal action. Then in the advection-free region, where $d(\delta p) / d p$ vanishes, we have from (24)

$$
\left(T-\frac{\kappa}{p}-\frac{\partial T}{\partial p}\right) \delta p=\partial T \text {. }
$$

Now in case of dry adiabatic cnange

$$
T^{\prime} \frac{\kappa}{p}-\frac{\partial T}{\partial p}=\left(\frac{\partial T}{\partial p}\right)_{\theta}-\frac{\partial T}{\partial p}
$$

where $\left(\frac{\partial T}{\partial p}\right)_{\theta}$ designate; the dry adiabatic temperature change with the pressure. Hence $\delta p$ is given by the ratio $\overline{A B} / \overline{C A}$ multiplied into $A$ as shown in Fig. 3. If the ratio is constant for

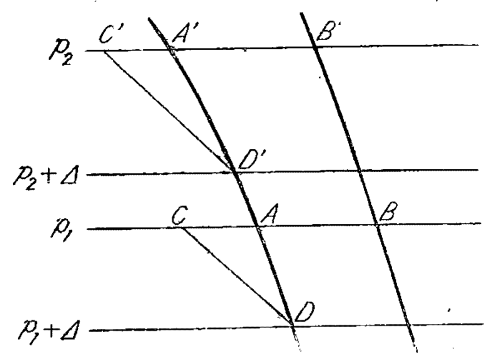

Fig. 3 Graphical method of finding advection-free region. 
every level within a certain region, the region advection-free. If not, the region is not advection-free, and the advection function $\delta p$ is not given by this ratio. If this ratio increases upwards, the advection function also increases upwards (so to speak, the region of positive advection) and vice versa.

\section{References}

[1] Rossby, C. G. 1928: Studies on the dynamics of the stratosphere, Beitr. z. Phys. d. fr. Atro. 41, p. 240.

[2] FRter, H. and II, S. 1935: Der Advectionsechanismus der atmosphärischen Druckschwankungen, ZS. Phys. 94, p. 662. Die Berechnung der Advektion, Met. ZS. 52, 1. 356.

[3] Ertes, H. 1938 : Methodel. und Probleme der dynamischen Meteorologie, p. 109.

[4] Rossby, C. G., 1927: Zustandsänderungen in atmosphärischen Tuftsäulen, Beitr. z. Phys. d. fr. Atin. 13, p. 163.

[5] Kuo Hsrao-LAN 1946: The mechanism of advection, Metneir of the Nat, Res. Inst. Met. Sinica 14, 1, p. 7 .

[6] Phimpps, H. 1939: Die Abweichung vom geostrophischen Wind, Met. ZS. 56, p. 460.

[7] Dufour, I. 1943 : L.es variations de la pression, de la teinpérature et du gradient ther mique vertical dans l'atmosphere, Inst. Roy. Nét. de Belgique. Missc. Fasc. 14, p. 14.

[8] ERTeL, H. 1936: Singuläre Advektion, Met. ZS. 53, p. 280. 